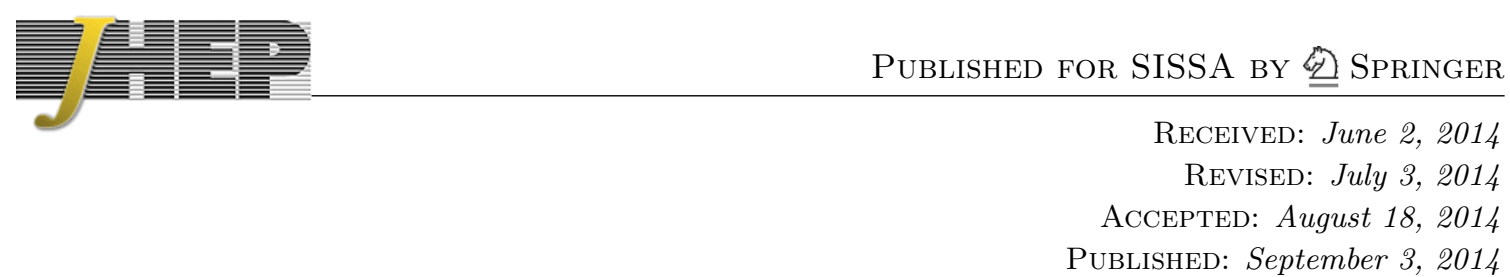

\title{
Static BPS black holes in U(1) gauged supergravity
}

\author{
Stefanos Katmadas \\ Dipartimento di Fisica, Università di Milano-Bicocca, I-20126 Milano, Italy \\ INFN, sezione di Milano-Bicocca, Milano, Italy \\ E-mail: stefanos.katmadas@unimib.it
}

ABSTRACT: We consider the flow equations for 1/4-BPS asymptotically $\mathrm{AdS}_{4}$ static black holes in Fayet-Iliopoulos gauged supergravity, using very special geometry identities to obtain a simplified form in the most general case. Under mild assumptions on the form of the solution, we analyse the flow equations and find an explicit solution for arbitrary gauging and charge vectors. Comparing with the corresponding attractor equations, we find that the solution is given in terms of exactly the same vector of parameters, implying that all regular attractors can be extended to full black hole solutions. We present explicit examples of black hole solutions with all complex scalars and allowed charges turned on, within the STU model and its truncations.

KeYwords: Black Holes in String Theory, Supergravity Models

ArXiv EPRINT: 1405.4901

Dedicated to the memory of Christos Katmadas 


\section{Contents}

1 Introduction $\quad 1$

2 Revisiting BPS attractors in $\mathrm{AdS}_{4} \quad 2$

3 Asymptotically AdS $_{4}$ BPS black holes 5

3.1 Analysis of the flow equations 5

$\begin{array}{ll}3.2 & \text { Reparametrisation of the radius }\end{array}$

$\begin{array}{ll}3.3 \text { Summary of static } \mathrm{AdS}_{4} \text { BPS solutions and the attractor limit } & 10\end{array}$

4 Explicit examples $\quad 11$

4.1 The $t^{3}$ model 13

$\begin{array}{lll}4.2 & \text { The STU model } & 14\end{array}$

5 Conclusion and outlook $\quad 16$

$\begin{array}{ll}\text { A Conventions on } \mathrm{N}=2 \text { supergravity } & 17\end{array}$

B Identities involving the quartic invariant $\quad 19$

\section{Introduction}

Supersymmetric backgrounds in supergravity have been a versatile tool for furthering the understanding of various aspects of string models and supersymmetric theories of gravity. Despite the rather strong constraints imposed on them, supersymmetric solutions very often provide a rich subsector of a supergravity theory that is at the same time simple enough to be studied analytically, granting good control over various quantities that are otherwise difficult to study. When specified to black holes embedded in flat spacetime, there is a long list of important results, based on the classification of supersymmetric solutions in both four and five dimensions [1-4], including various novel solutions and important insights in the dual string theoretic picture. Unlike the asymptotically flat solutions, there is much less known about BPS solutions with AdS asymptotics, as a general classification is missing. The known solutions include various black hole and black brane solutions that asymptote either to $\mathrm{AdS}_{4}$ or $\mathrm{AdS}_{5}$, that have been obtained by various methods.

In particular, BPS black hole solutions in $\mathrm{AdS}_{4}$ [5-7], have been the subject of considerable recent interest, starting with the first $\mathrm{AdS}_{4}$ solution with a spherical horizon, obtained in [8], which was later expanded upon with the work of [9-18]. These extensions include various analytical and numerical solutions, mainly describing static backgrounds, as well as some notable stationary solutions. A common feature of all these BPS solutions 
in $\mathrm{AdS}_{4}$ is the fact that the scalars are usually restricted to special configurations throughout the spacetime, in order to render the equations tractable. Indeed, almost all the known solutions are given for gaugings that allow for vanishing axions at infinity, which are then assumed to vanish everywhere, so that only half of the BPS flow equations are relevant.

In this paper we consider the extension of these results to the most general case, for static 1/4-BPS black holes in Fayet-Iliopoulos (FI) gauged supergravity with symmetric scalar manifold. Using techniques of very special geometry, we show that the BPS flow equations can be drastically simplified, so that they involve only one combination of the scalars, which in addition transforms covariantly under electric/magnetic duality reparametrisations.

The resulting equations are again nonlinear and therefore more complicated than the corresponding ones in the ungauged theory [2]. Nevertheless, they allow for much better analytic control for general gaugings and scalar configurations. Employing a general expansion in powers of the radial coordinate in $\mathrm{AdS}_{4}$, we obtain a very restricted system that precisely corresponds to the standard ansatz of $[8-10,17,18]$, but allows for a general flow of the complex scalars. The latter is parametrised in terms of a single symplectic vector, which is subject to a number of constraints and is determined by the gauging and the electromagnetic charges.

As already noted in $[9,10,15,17,18]$, the charges are in fact not completely free, but are restricted for a given gauging vector. In order to systematically study the possible solutions, we recast the attractor equations in terms of a single real symplectic vector that describes both the values of the scalar fields and the charges. Upon comparison with the vector parametrising the general asymptotically $\mathrm{AdS}_{4}$ solution, we find the two vectors to be exactly equivalent, implying that any regular attractor geometry in the standard branch $^{1}$ can be extended to a full black hole geometry.

This paper is organised as follows. In section 2 we revisit the attractor geometry in $\mathrm{AdS}_{4}$, recasting the attractor equations in a form that is particularly well suited for the comparison with the full flow. In addition, this provides a simple example of the technique used in the discussion of the full BPS equations. The latter is presented in section 3, where we recast the BPS equations in a form that can be analysed by elementary methods without the need of assumptions on the form of the gauging or scalars. Section 4 is devoted to a number of explicit examples of black holes with both electric and magnetic charges turned on, within the STU model and its truncations. We conclude in section 5, where we discuss possible extensions of the results of this paper. The two appendices give the basic conventions used throughout the paper and some very useful identities satisfied by the quartic invariant of very special geometry.

\section{Revisiting BPS attractors in $\mathrm{AdS}_{4}$}

In this section we revisit the BPS attractor equations in FI gauged supergravity [6, 9, 15, 19, 20], recasting the symplectic covariant equations in a form that can be easily connected to the flow equations for the full asymptotically $\mathrm{AdS}_{4}$ black hole geometries. Our analysis is

\footnotetext{
${ }^{1}$ As explained in section 2 , there are two branches of attractors, only one of which appears to be relevant.
} 
based only on symplectic vectors in the real basis and is therefore somewhat complimentary to the corresponding analysis of [15], which relied on the complex basis.

The starting point is the expressions for the metric and (constant) scalar fields at the $\mathrm{AdS}_{2} \times \mathrm{S}^{2}$ attractor, which take the form

$$
\begin{aligned}
& d s^{2}=-\mathrm{e}^{2 U_{0}} r^{2} d t^{2}+\mathrm{e}^{-2 U_{0}} \frac{d r^{2}}{r^{2}}+\mathrm{e}^{2\left(\psi_{0}-U_{0}\right)}\left(d \theta^{2}+\sin ^{2} \theta d \phi^{2}\right), \\
& 2 \mathrm{e}^{2 \psi_{0}-U_{0}} \operatorname{Im}\left(\mathrm{e}^{-\mathrm{i} \alpha} \mathcal{V}\right)=\Gamma+\mathrm{e}^{2\left(\psi_{0}-U_{0}\right)} \mathrm{J} G .
\end{aligned}
$$

Here, $\Gamma$ is the vector of charges, which completely fixes the field strengths in the static case through (A.1), while $G$ is a symplectic vector of electric and magnetic FI terms and $\mathrm{J}$ is the scalar dependent complex structure defined in appendix A. The positive constants $\mathrm{e}^{U_{0}}$ and $\mathrm{e}^{\psi_{0}}$ control the radii of $\mathrm{AdS}_{2}$ and $\mathrm{S}^{2}$ respectively and have been chosen for convenience in connecting with the full flow in later sections. The phase $\mathrm{e}^{-\mathrm{i} \alpha}$ is such that the central charges $^{2}$ of the charge, $Z(\Gamma)$, and the gauging, $Z(G)$, satisfy

$$
-\mathrm{e}^{2\left(U_{0}-\psi_{0}\right)} \operatorname{Re}\left(\mathrm{e}^{-\mathrm{i} \alpha} Z(\Gamma)\right)=\operatorname{Im}\left(\mathrm{e}^{-\mathrm{i} \alpha} Z(G)\right)=\frac{1}{2} \mathrm{e}^{U_{0}},
$$

while its complex conjugate is also trivially satisfied, due to the additional conditions

$$
\begin{aligned}
\operatorname{Im}\left(\mathrm{e}^{-\mathrm{i} \alpha} Z(\Gamma)\right) & =\operatorname{Re}\left(\mathrm{e}^{-\mathrm{i} \alpha} Z(G)\right)=0, \\
\langle G, \Gamma\rangle & =-1 .
\end{aligned}
$$

The latter originate from the fact that the BPS equations relate these quantities to the (vanishing) Kähler connection and the spin connection respectively.

In order to characterise the solution to the above equations, we start by parametrising the symplectic section in a convenient way in terms of a vector, $\mathcal{B}$, as

$$
2 \mathrm{e}^{\psi_{0}-U_{0}} \operatorname{Im}\left(\mathrm{e}^{-\mathrm{i} \alpha} \mathcal{V}\right)=\mathcal{B},
$$

and solve for the second of (2.3), so that $\psi_{0}$ and the section become

$$
\begin{aligned}
2 \mathrm{e}^{-U_{0}} \operatorname{Im}\left(\mathrm{e}^{-\mathrm{i} \alpha} \mathcal{V}\right) & =\langle G, \mathcal{B}\rangle^{-1} \mathcal{B}, \\
\mathrm{e}^{\psi_{0}} & =\langle G, \mathcal{B}\rangle .
\end{aligned}
$$

In terms of $\mathcal{B}$, the scale factor and the real part of the section are given by

$$
\mathrm{e}^{-4 U_{0}}=\langle G, \mathcal{B}\rangle^{-4} I_{4}(\mathcal{B}), \quad 2 \mathrm{e}^{U_{0}} \operatorname{Re}\left(\mathrm{e}^{-\mathrm{i} \alpha} \mathcal{V}\right)=\frac{1}{2}\langle G, \mathcal{B}\rangle I_{4}(\mathcal{B})^{-1} I_{4}^{\prime}(\mathcal{B}) .
$$

The next step is to insert this vector in the (2.2) to obtain an equation for the vector $\mathcal{B}$, which requires an expression for the action of the scalar dependent operator $\mathrm{J}$ on the vector of gauging, $G$, in terms of $\mathcal{B}$. As explained in appendix B, this can be done using equation (B.9), which when evaluated for $G$, reads

$$
\frac{1}{2} I_{4}^{\prime}(\mathcal{B}, \mathcal{B}, G)=2\langle G, \mathcal{B}\rangle \mathcal{B}-2\langle G, \mathcal{B}\rangle^{2} \mathrm{e}^{-2 U_{0}} \mathrm{~J} G
$$

\footnotetext{
${ }^{2}$ See (A.7) for a general definition of central charges.
} 
where we used the definition (2.7) and (2.4). Inserting this in (2.2) we obtain

$$
\frac{1}{4} I_{4}^{\prime}(\mathcal{B}, \mathcal{B}, G)=\Gamma
$$

which can be solved to find the vector $\mathcal{B}$ in terms of the gauging and the charge (see section 4 for example solutions). The scalar fields and metric components are then given by $(2.7),(2.9)$ and (2.8) respectively. Note that all BPS equations are satisfied except (2.4)(2.5), which lead to the constraints

$$
\langle\mathcal{B}, \Gamma\rangle=I_{4}(\mathcal{B}, \mathcal{B}, \mathcal{B}, G)=0, \quad \frac{1}{4} I_{4}(\mathcal{B}, \mathcal{B}, G, G)=-1 .
$$

Note that the two equations in (2.4) have become linearly dependent due to (2.11), in line with the fact that only one of them fixes a physical phase, the second one being the unphysical phase $\alpha$. Therefore, (2.12) reduce the $2 n_{\mathrm{v}}+2$ components of $\mathcal{B}$ to $2 n_{\mathrm{v}}$ independent components. Finally, the black hole entropy, computed by the horizon area is given by the simple expression

$$
\mathcal{E}=\pi \sqrt{I_{4}(\mathcal{B})} .
$$

Note that this expression is reminiscent of the entropy formula for asymptotically flat extremal solutions, which is obtained from (2.13) by replacing $\mathcal{B}$ by the charge vector $\Gamma$.

For future reference, we briefly discuss a more direct formula for the entropy, involving only charges and gaugings, obtained in [15] by rearranging (2.2)-(2.4) into the following relation

$$
\Gamma+\mathrm{i} \mathrm{e}^{2\left(\psi_{0}-U_{0}\right)} G=\mathrm{i} \bar{Z}(\Gamma) \mathcal{V}-\mathrm{i} g^{\bar{i} j} \bar{Z}_{\bar{\imath}}(\Gamma) D_{j} \mathcal{V} .
$$

The last relation is purely holomorphic in the the symplectic section and its derivatives, so that one directly obtains from (B.7) that

$$
I_{4}^{\prime}\left(\Gamma+\mathrm{i} \mathrm{e}^{2\left(\psi_{0}-U_{0}\right)} G\right)=\frac{1}{6} c_{i j k} \bar{Z}^{i} \bar{Z}^{j} \bar{Z}^{k} \mathcal{V}, \quad I_{4}\left(\Gamma+\mathrm{i} \mathrm{e}^{2\left(\psi_{0}-U_{0}\right)} G\right)=0 .
$$

These are solved by the above expressions in terms of $\mathcal{B}$, but the second can be easily solved for the radius of the sphere $\mathrm{as}^{3}$

$$
\begin{aligned}
\mathrm{e}^{4\left(\psi_{0}-U_{0}\right)}=I_{4}(\mathcal{B}) & =\frac{1}{2 I_{4}(G)}\left(\frac{1}{4} I_{4}(G, G, \Gamma, \Gamma) \pm \sqrt{\frac{1}{16} I_{4}(G, G, \Gamma, \Gamma)^{2}-4 I_{4}(G) I_{4}(\Gamma)}\right) \\
I_{4}(G, G, G, \Gamma) & =I_{4}(G, \Gamma, \Gamma, \Gamma)=0
\end{aligned}
$$

where only the plus sign leads to a positive radius for all examples we discuss in section 4 . The first of these arises from the real part of (2.15) and provides the required entropy formula, while (2.17) arises from the imaginary part and represents a constraint on the

\footnotetext{
${ }^{3}$ Note that there exists another branch of solutions, for which $\mathrm{e}^{4\left(\psi_{0}-U_{0}\right)} \sim I_{4}(G, \Gamma, \Gamma, \Gamma) / I_{4}(G, G, G, \Gamma)$, see [15] for details. Here, we display only the branch that arises from the BPS equations in the bulk, as derived in the next section. It is an interesting problem to find physically relevant situations where this second branch arises.
} 
charges, since the two conditions are linearly related upon using the result (2.11) above, together with the identities presented in appendix B.

As will be shown in the next section, the flow to asymptotic $\mathrm{AdS}_{4}$ imposes (2.17), so that this additional condition is necessary for extending the attractor to a full black hole geometry. In fact, the treatment of the next section allows for the full solution to be constructed if the attractor solution for the scalars is known, in exactly the same way as for asymptotically flat black holes.

Obtaining the attractor solution involves solving (2.11), which is a complicated task in general, since it corresponds to a $2\left(n_{\mathrm{v}}+1\right)$ dimensional system of quadratic equations, on which three constraints have to be imposed. We have two constraints from (2.12) and an additional one from (2.17), reducing $\mathcal{B}$, and therefore also $\Gamma$, to $2 n_{\mathrm{v}}-1$ independent components. For restricted cases, it is possible to solve this system for all symmetric models, see [17] for a closely related computation. ${ }^{4}$ In section 4 we present explicit solutions to the STU model and its truncations. The derivation of a general solution for $\mathcal{B}$ in terms of the gauging and the charge vector falls outside the scope of this work.

\section{$3 \quad$ Asymptotically AdS $_{4}$ BPS black holes}

In this section, we consider the full asymptotically $\mathrm{AdS}_{4}$ flow for $1 / 4-\mathrm{BPS}$ static black holes, generalising the analysis of the attractor geometry presented in the previous section. This is based on the static flow equations as derived in [9], whose conventions we follow up to some changes in naming.

\subsection{Analysis of the flow equations}

For the class of solutions we are interested in, the appropriate ansatz for a static metric is

$$
d s^{2}=-\mathrm{e}^{2 U} d t^{2}+\mathrm{e}^{-2 U}\left(d r^{2}+\mathrm{e}^{2 \psi} d \theta^{2}+\mathrm{e}^{2 \psi} \sin ^{2} \theta d \phi^{2}\right),
$$

which allows for a non-flat three dimensional base. In these variables, the boundary conditions for the metric fields at infinity are given by

$$
\mathrm{e}^{\psi}=I_{4}(G)^{1 / 4} r^{2}+\mathcal{O}(r), \quad \mathrm{e}^{U}=I_{4}(G)^{1 / 4} r+\mathcal{O}\left(r^{0}\right),
$$

where we used the requirement that $R_{\mathrm{AdS}}=I_{4}(G)^{-1 / 4}$ is the radius of the asymptotic $\mathrm{AdS}_{4}$. The BPS flow equations are [9]:

$$
\begin{aligned}
2 e^{2 \psi}\left(e^{-U} \operatorname{Im}\left(e^{-i \alpha} \mathcal{V}\right)\right)^{\prime}+e^{2(\psi-U)} \mathrm{J} G & +4 e^{2 \psi-U}\left(Q_{r}+\alpha^{\prime}\right) \operatorname{Re}\left(e^{-i \alpha} \mathcal{V}\right)+\Gamma=0, \\
Q_{r}+\alpha^{\prime} & =-2 e^{-U} \operatorname{Re}\left(e^{-i \alpha} Z(G)\right) \\
\psi^{\prime} & =2 e^{-U} \operatorname{Im}\left(e^{-i \alpha} Z(G)\right)
\end{aligned}
$$

where the charge and gauging are required to satisfy the quantisation condition

$$
\langle G, \Gamma\rangle=-1 .
$$

\footnotetext{
${ }^{4}$ Note that the vector $\mathcal{B}$ used here is related to the one used in [17] by a shift of the radial coordinate, cf. (3.30) and the relevant discussion below.
} 
In writing these equations, we used the definition of the central charge functions in (A.7), which depend on the scalar fields and are defined everywhere in spacetime, rather than the central charge defined at infinity.

We now proceed to recast these BPS equations in a simpler form, so that they depend on the scalars only through the imaginary part of the section. Similar to the analysis of the attractor, we use again the identity (B.9) to express the action of the complex structure, $\mathrm{J}$, on the gauging as

$$
\begin{aligned}
\frac{1}{4} I_{4}^{\prime}\left(2 \operatorname{Im}\left(e^{-i \alpha} \mathcal{V}\right), 2 \operatorname{Im}\left(e^{-i \alpha} \mathcal{V}\right), G\right)= & -\mathrm{J} G+4 \operatorname{Im}\left(e^{-i \alpha} Z(G)\right) \operatorname{Im}\left(e^{-i \alpha} \mathcal{V}\right) \\
& +8 \operatorname{Re}\left(e^{-i \alpha} Z(G)\right) \operatorname{Re}\left(e^{-i \alpha} \mathcal{V}\right)
\end{aligned}
$$

Using (3.4) to rewrite the flow equation for the section as

$$
2 \mathrm{e}^{2 \psi}\left(\mathrm{e}^{-U} \operatorname{Im}\left(\mathrm{e}^{-i \alpha} \mathcal{V}\right)\right)^{\prime}+e^{2(\psi-U)} \mathrm{J} G-8 \mathrm{e}^{2(\psi-U)} \operatorname{Re}\left(e^{-i \alpha} Z(G)\right) \operatorname{Re}\left(\mathrm{e}^{-i \alpha} \mathcal{V}\right)+\Gamma=0,
$$

and comparing with (3.7), we find the following simplified flow equation, where the scalar fields appear solely through the symplectic section

$$
\begin{aligned}
& 2 \mathrm{e}^{2 \psi}\left(\mathrm{e}^{-U} \operatorname{Im}\left(\mathrm{e}^{-i \alpha} \mathcal{V}\right)\right)^{\prime}+4 e^{2(\psi-U)} \operatorname{Im}\left(e^{-i \alpha} Z(G)\right) \operatorname{Im}\left(e^{-i \alpha} \mathcal{V}\right) \\
& -\frac{1}{4} e^{2(\psi-U)} I_{4}^{\prime}\left(2 \operatorname{Im}\left(e^{-i \alpha} \mathcal{V}\right), 2 \operatorname{Im}\left(e^{-i \alpha} \mathcal{V}\right), G\right)+\Gamma=0
\end{aligned}
$$

The first two terms can be combined upon use of (3.5) to obtain

$$
2 \mathrm{e}^{\psi}\left(\mathrm{e}^{\psi-U} \operatorname{Im}\left(\mathrm{e}^{-i \alpha} \mathcal{V}\right)\right)^{\prime}-\frac{1}{4} e^{2(\psi-U)} I_{4}^{\prime}\left(2 \operatorname{Im}\left(e^{-i \alpha} \mathcal{V}\right), 2 \operatorname{Im}\left(e^{-i \alpha} \mathcal{V}\right), G\right)+\Gamma=0,
$$

which, together with (3.5) form a system of first order equations for the quantities $\mathrm{e}^{\psi}$ and

$$
2 \mathrm{e}^{\psi-U} \operatorname{Im}\left(\mathrm{e}^{-\mathrm{i} \alpha} \mathcal{V}\right) \equiv \mathcal{H}
$$

alone, as

$$
\begin{aligned}
\left(\mathrm{e}^{\psi}\right)^{\prime} & =\langle G, \mathcal{H}\rangle, \\
\mathrm{e}^{\psi} \mathcal{H}^{\prime}-\frac{1}{4} I_{4}^{\prime}(\mathcal{H}, \mathcal{H}, G)+\Gamma & =0 .
\end{aligned}
$$

Despite the non-linearity of (3.13), it is simple to find solutions to this system, using as input the fact that $\mathrm{e}^{\psi}$ is a regular function which behaves $\sim r^{2}$ asymptotically and has a single zero at the horizon. Since the first of (3.12) is linear, the vector $\mathcal{H}$ must be at most linear in the radial coordinate. One may then consider an expansion consistent with the standard asymptotic expansion in $\mathrm{AdS}_{4}$, as

$$
\mathcal{H}=\mathcal{A} r+\mathcal{B}+\sum_{n \geq 1} \mathcal{C}_{n} \frac{1}{r^{n}}
$$

where $\mathcal{A}, \mathcal{B}$ and the $\mathcal{C}_{n}$ for $n \geq 1$ are constant vectors. For simplicity, we set all the vectors $\mathcal{C}_{n}=0$ for the moment and perform the analysis for the linear terms only, postponing the 
justification of this truncation at the end of this subsection. The equation for $\mathrm{e}^{\psi}$ can now be easily integrated as

$$
\begin{aligned}
\langle G, \mathcal{H}\rangle & =2 r I_{4}(G)^{1 / 4}+\langle G, \mathcal{B}\rangle, \\
\mathrm{e}^{\psi} & =I_{4}(G)^{1 / 4} r^{2}+\langle G, \mathcal{B}\rangle r+c,
\end{aligned}
$$

where $c$ is an arbitrary constant and we used (3.2) to determine the coefficient of the leading term in $\mathrm{e}^{\psi}$.

We now consider each of the three types of terms arising in (3.13) upon using (3.15), namely constant, linear and quadratic in the radial coordinate, $r$. The quadratic terms lead to

$$
I_{4}(G)^{1 / 4} \mathcal{A}-\frac{1}{4} I_{4}^{\prime}(\mathcal{A}, \mathcal{A}, G)=0 \quad \Rightarrow \quad \mathcal{A}=\frac{1}{2} I_{4}(G)^{-3 / 4} I_{4}^{\prime}(G)
$$

which is the natural combination homogeneous in $G$ and agrees with the result of [17]. The terms linear in $r$ lead to

$$
\langle G, \mathcal{B}\rangle \mathcal{A}-\frac{1}{2} I_{4}^{\prime}(\mathcal{A}, \mathcal{B}, G)=0 \quad \Rightarrow \quad\langle\mathcal{A}, \mathcal{B}\rangle=0
$$

where we used the explicit form of $\mathcal{A}$ in (3.16) and (B.6) to obtain the second equality. Finally, the constant part of (3.13) reads

$$
c \mathcal{A}-\frac{1}{4} I_{4}^{\prime}(\mathcal{B}, \mathcal{B}, G)+\Gamma=0
$$

which determines $\mathcal{B}$ and $c$ in terms of the charges and the gauging. Taking the inner product with $G$, we obtain

$$
c=\frac{1}{2} I_{4}(G)^{-1 / 4}\left(-\langle G, \Gamma\rangle+\frac{1}{4} I_{4}(\mathcal{B}, \mathcal{B}, G, G)\right),
$$

while the inner product of (3.18) with $\mathcal{A}$ and $\mathcal{B}$ leads to

$$
\langle\mathcal{A}, \Gamma\rangle=0, \quad\langle\mathcal{B}, \Gamma\rangle=\frac{1}{4} I_{4}(\mathcal{B}, \mathcal{B}, \mathcal{B}, G),
$$

respectively, where we used (3.17) to obtain the first result.

The final equation to be imposed is the condition (3.4), so we compute each term individually

$$
\begin{aligned}
Q_{r}+\alpha^{\prime} & =-\frac{1}{2} \mathrm{e}^{2(U-\psi)}\langle\mathcal{A}, \mathcal{B}\rangle=0, \\
2 e^{U} \operatorname{Re}\left(e^{-i \alpha} W\right) & =\frac{1}{2} \mathrm{e}^{\psi} I_{4}(\mathcal{H})^{-1}\left\langle G, I_{4}^{\prime}(\mathcal{H})\right\rangle \\
& =\frac{1}{2} \mathrm{e}^{\psi} I_{4}(\mathcal{H})^{-1}\left(\langle G, \mathcal{B}\rangle\langle\mathcal{B}, \mathcal{A}\rangle r+\frac{1}{6} I_{4}(G, \mathcal{B}, \mathcal{B}, \mathcal{B})\right) \\
& =\frac{1}{12} \mathrm{e}^{\psi} I_{4}(\mathcal{H})^{-1} I_{4}(\mathcal{B}, \mathcal{B}, \mathcal{B}, G),
\end{aligned}
$$


where (2.9) was used for the real part of the section and (3.17) was used repeatedly to simplify the result. We therefore find that (3.4) is satisfied if we set

$$
I_{4}(\mathcal{B}, \mathcal{B}, \mathcal{B}, G)=\langle\mathcal{B}, \Gamma\rangle=0
$$

where the second equality follows from (3.20) above. This concludes our analysis of the BPS equations.

Finally, we return to the more general ansatz in (3.14) and give some evidence for the exclusion of any terms of negative power in $r$ in that expansion. We thus consider a general vector $\mathcal{H}$ and analyse the horizon behaviour of the flow equation (3.13) and its derivatives, corresponding to a Taylor expansion of the type

$$
\mathcal{H}=\sum \frac{1}{n !} \mathcal{H}_{0}^{(n)}\left(r-r_{0}\right)^{n}=\mathcal{H}_{0}+\mathcal{H}_{0}^{(1)}\left(r-r_{0}\right)+\frac{1}{2} \mathcal{H}_{0}^{(2)}\left(r-r_{0}\right)^{2}+\frac{1}{6} \mathcal{H}_{0}^{(3)}\left(r-r_{0}\right)^{3}+\ldots
$$

where we denote horizon values by the 0 subscript and $\mathcal{H}_{0}^{(n)}$ is the $n$-th derivative at the horizon, which we take to be at $r=r_{0}$. The latter is by definition the solution of $\mathrm{e}^{\psi}=0$, where (3.13) becomes identical to (2.11), as

$$
\Gamma=\frac{1}{4} I_{4}^{\prime}\left(\mathcal{H}_{0}, \mathcal{H}_{0}, G\right) .
$$

Similarly, taking the derivative of (3.13) and evaluating at the horizon, one finds the eigenvalue equation

$$
\left\langle G, \mathcal{H}_{0}\right\rangle \mathcal{H}_{0}^{(1)}-\frac{1}{2} I_{4}^{\prime}\left(\mathcal{H}_{0}^{(1)}, \mathcal{H}_{0}, G\right)=0 \quad \Rightarrow \quad \mathcal{H}_{0}^{(1)}=\mathcal{A} .
$$

where $\mathcal{A}$ is as in (3.16), which matches the derivative of $\mathcal{H}$ at infinity. In order to obtain this result, we used (B.6) and the requirement $\left\langle\mathcal{H}_{0}, \mathcal{H}_{0}^{(1)}\right\rangle=0$, which follows from (3.4) at the horizon. Continuing with the higher derivatives of (3.13) evaluated at the horizon, one finds an eigenvalue equation involving the same operator as in (3.26), for the various derivatives of $\mathcal{H}$. For example, the second derivative leads to

$$
2\left\langle G, \mathcal{H}_{0}\right\rangle \mathcal{H}_{0}^{(2)}-\frac{1}{2} I_{4}^{\prime}\left(\mathcal{H}_{0}^{(2)}, \mathcal{H}_{0}, G\right)=0
$$

where we used (3.26) to simplify the result, while the third derivative is given by the eigenvalue equation

$$
\begin{aligned}
& 3\left\langle G, \mathcal{H}_{0}\right\rangle \mathcal{K}^{(3)}-\frac{1}{2} I_{4}^{\prime}\left(\mathcal{K}^{(3)}, \mathcal{H}_{0}, G\right)=0 \\
& \mathcal{K}^{(3)}=\mathcal{H}_{0}^{(3)}+3 \frac{\left\langle G, \mathcal{H}_{0}^{(1)}\right\rangle}{\left\langle G, \mathcal{H}_{0}\right\rangle} \mathcal{H}_{0}^{(2)}-\frac{\left\langle G, \mathcal{H}_{0}^{(2)}\right\rangle}{4\left\langle G, \mathcal{H}_{0}\right\rangle} \mathcal{H}_{0}^{(1)}
\end{aligned}
$$

Therefore, (3.27)-(3.28) demand the existence of two eigenvalues for the matrix $I_{4}\left(\mathcal{H}_{0}, G\right)$ that are twice and three times as large as the one in (3.26) respectively. This is not true in general, but leads to constraints on the vector $\mathcal{H}_{0}$ and therefore the charges through (3.25). In particular, since the matrix involved is symplectic, the corresponding negative eigen 
values must also be present, so that there are four conditions resulting from (3.27)-(3.28). By consistency, one further obtains the three conditions

$$
\left\langle\mathcal{H}_{0}^{(1)}, \mathcal{H}_{0}^{(2)}\right\rangle=\left\langle\mathcal{H}_{0}^{(1)}, \mathcal{H}_{0}^{(3)}\right\rangle=\left\langle\mathcal{H}_{0}^{(3)}, \mathcal{H}_{0}^{(2)}\right\rangle=0,
$$

which further constrain the allowed eigenvectors.

Using exactly the same procedure, one can show that this pattern continues to higher orders, so that the same operator in (3.26)-(3.28) must have integer spaced eigenvalues, thus constraining the charges further. We conclude that adding nonlinear terms to $\mathcal{H}$, as the $\mathcal{C}_{n}$ in (3.14), is not allowed in general, as that would turn on an arbitrary number of derivatives of $\mathcal{H}$ at the horizon that cannot be accommodated by the finite dimensional matrix above. One may still hope to find a solution for restricted charges, by arranging that the various nonlinear terms cancel each other at the horizon. Moreover, a more general expansion at the horizon still remains to be done in principle, allowing for non-integer powers of the radial variable. However, nonlinear terms in $\mathcal{H}$ are inconsistent with the shift invariance of the equations along $r$ discussed in section 3.2 below, as well as with all known asymptotically $\mathrm{AdS}_{4}$ black hole solutions, both extremal and non-extremal alike, in which these terms do not appear, see e.g. [21, 22] for an overview of the relevant ansatze.

\subsection{Reparametrisation of the radius}

It is interesting to note a redundancy of the above equations, that will prove useful in the discussion of explicit examples. It is simple to check using (3.18), combined with (3.16)(3.17), that the shift

$$
\mathcal{B} \rightarrow \mathcal{B}+b \mathcal{A},
$$

for any constant, $b$, can be reabsorbed by a shift of the radial coordinate, $r$, both from the scalars and the metric. For example, shifts in $\langle G, \mathcal{B}\rangle$ and $c$, which are linear and quadratic in $b$ respectively, are such that $(3.15)$ becomes

$$
\mathrm{e}^{\psi}=I_{4}(G)^{1 / 4}(r+b)^{2}+\langle G, \mathcal{B}\rangle(r+b)+c .
$$

Turning the argument around, one can always shift the radial variable so as to arrange that (3.30) leads to

$$
\langle G, \mathcal{B}\rangle=0
$$

holds, which is the choice usually taken in the literature.

Alternatively, one may use this freedom to impose $c=0$. In this case, we find

$$
\mathrm{e}^{\psi}=\left(I_{4}(G)^{1 / 4} r+\langle G, \mathcal{B}\rangle\right) r,
$$

where we assume without loss of generality that

$$
\langle G, \mathcal{B}\rangle>0
$$

since one may always shift the nonzero root of $\mathrm{e}^{\psi}$ to $\pm\langle G, \mathcal{B}\rangle$. In contradistinction with the previous choice, this inner product is not allowed to vanish in order to have a well defined horizon. Setting $c=0$ leads to a simplification of (3.18), but most importantly it makes the horizon limit clear, as it is always located at $r=0$ by construction. We will use this second choice for the remainder of this paper, for simplicity. 


\subsection{Summary of static $\mathrm{AdS}_{4}$ BPS solutions and the attractor limit}

For the convenience of the reader we provide a summary of the general duality covariant equations describing static $\mathrm{AdS}_{4} 1 / 4$-BPS solutions. The metric is given by

$$
\begin{aligned}
d s^{2} & =-\mathrm{e}^{2 U} d t^{2}+\mathrm{e}^{-2 U}\left(d r^{2}+\mathrm{e}^{2 \psi} d \theta^{2}+\mathrm{e}^{2 \psi} \sin ^{2} \theta d \phi^{2}\right), \\
\mathrm{e}^{\psi} & =\left(I_{4}(G)^{1 / 4} r+\langle G, \mathcal{B}\rangle\right) r .
\end{aligned}
$$

The scale factor $\mathrm{e}^{2 U}$ and the scalar fields are given by

$$
2 \mathrm{e}^{-U} \operatorname{Im}\left(\mathrm{e}^{-\mathrm{i} \alpha} \mathcal{V}\right)=\mathrm{e}^{-\psi}(\mathcal{A} r+\mathcal{B}), \quad \mathcal{A}=\frac{1}{2} I_{4}(G)^{-3 / 4} I_{4}^{\prime}(G),
$$

where we note that the asymptotic value of both are completely fixed by the gaugings, through the vector, $\mathcal{A}$, as expected. The attractor flow is governed by the vector, $\mathcal{B}$, which is fixed in terms of the charge through

$$
\frac{1}{4} I_{4}^{\prime}(\mathcal{B}, \mathcal{B}, G)=\Gamma
$$

Note that there are some conditions on both $\mathcal{B}$ and $\Gamma$ that arise both from supersymmetry and from imposing consistency of the above equations and read

$$
\begin{aligned}
& \langle\mathcal{A}, \Gamma\rangle=\langle\mathcal{A}, \mathcal{B}\rangle=\langle\Gamma, \mathcal{B}\rangle=0, \\
& \langle G, \Gamma\rangle=-\kappa, \\
& \langle G, \mathcal{B}\rangle>0 .
\end{aligned}
$$

Here, we have introduced the constant, $\kappa$, generalising (3.6) to allow for solutions with different horizon geometry $[8,9]$. Setting $\kappa=1$ leads to black holes with a spherical horizon, whereas $\kappa=-1$ or $\kappa=0$ lead to black holes with a hyperbolic or flat horizons respectively.

At this point it is instructive to count the number of parameters allowed for the solutions above. Taking the gaugings to be arbitrary, (3.38) impose two conditions on each of $\Gamma$ and $\mathcal{B}$, reducing them to $2 n_{\mathrm{v}}$ components each. Note that this is consistent with (3.37), which now also contains the same number of independent components. The constraint (3.39) further reduces the number of free charges to $2 n_{\mathrm{v}}-1$, while it implies, through (3.37), that

$$
\frac{1}{4} I_{4}(\mathcal{B}, \mathcal{B}, G, G)=-1
$$

which can be viewed as a normalisation condition on the solutions of (3.37).

Comparing with asymptotically flat black holes, it is useful to note that (3.38) still holds in that case ${ }^{5}$ and expresses the requirement of vanishing NUT charge. Exactly as in the above analysis, the vector, $\mathcal{A}$, is again parametrising the asymptotic scalars. The latter are arbitrary in the ungauged theory, so $\mathcal{A}$ is also a priori arbitrary and is only constrained by (3.38). In the gauged theory however, the asymptotic scalars are completely fixed by

\footnotetext{
${ }^{5}$ In the asymptotically flat case, the vector $\mathcal{B}$ is not relevant.
} 
the gauging through (3.36), so that (3.38) instead becomes a constraint on the allowed charge.

Finally, we connect with the attractor analysis of section 2, by taking the near horizon limit. In the chosen coordinates this is located at $r=0$, so that one only needs to expand the above expressions for small $r$. The metric scale factors take the form

$$
\mathrm{e}^{\psi} \rightarrow\langle G, \mathcal{B}\rangle r \equiv \mathrm{e}^{\psi_{0}} r, \quad \mathrm{e}^{U} \rightarrow\langle G, \mathcal{B}\rangle I_{4}(\mathcal{B})^{-1 / 4} r \equiv \mathrm{e}^{U_{0}} r
$$

where we defined the constants $\mathrm{e}^{\psi_{0}}$ and $\mathrm{e}^{U_{0}}$. Similarly, the near horizon limit of the scalars leads to

$$
2 \mathrm{e}^{-U_{0}} \operatorname{Im}\left(\mathrm{e}^{-\mathrm{i} \alpha} \mathcal{V}\right)=\langle G, \mathcal{B}\rangle^{-1} \mathcal{B}
$$

These expressions are identical to (2.7), (2.8) and (2.9) derived at the attractor. Similarly, (3.37) matches with (2.11) and (3.38)-(3.39) impose the constraints (2.12) and (2.17). We conclude that any attractor solution satisfying to the conditions presented in section 2 can be extended to an asymptotically $\mathrm{AdS}_{4}$ black hole.

\section{Explicit examples}

In this section we consider some explicit solutions for three cubic models, namely the STU model and its two straightforward truncations, the two-modulus $s t^{2}$ model and the onemodulus $t^{3}$ model, which are the benchmark examples for all the symmetric models in the infinite $\mathrm{SO}(2, n+1)$ series. While our discussion is by no means exhaustive, we illustrate the salient features of BPS solutions, focusing on recovering the largest possible number of parameters. In particular, we recast the magnetic solutions of [8-10] in the conventions of this paper, so that the horizon is at a fixed locus $r=0$. In addition, we display explicit expressions for the dyonic solutions constructed recently in [18] by duality rotations on the magnetic solutions. While all the solutions for the STU model presented in the papers above and in the present section, should be limits of the general solution given recently in [21], this connection is difficult to implement explicitly.

The STU model is defined by the prepotential

$$
F=\frac{X^{1} X^{2} X^{3}}{X^{0}}
$$

and contains three physical complex scalar fields that appear completely symmetrically, each coming from a vector multiplet. In all examples in this section, we will choose the gauging vector, $G$ to be such that the axions vanish on the $\mathrm{AdS}_{4}$ vacuum at infinity. In particular, we take

$$
G=\left(g^{0}, 0,0,0, g_{1}, g_{2}, g_{3}, 0\right)^{T},
$$

for the STU model, while we reduce this vector by taking $g_{3}=g_{2}$ for the $s t^{2}$ model and $g_{3}=g_{2}=g_{1}$ for the $t^{3}$ model. Note that (4.2) can be easily rotated to an electric frame, at the expense of making (4.1) more complicated. Given this vector, the radius of $\mathrm{AdS}_{4}$ 
and the asymptotic scalars are controlled by the vector $\mathcal{A}$ in (3.36), which reads

$$
\mathcal{A}=\frac{1}{2} I_{4}(G)^{-3 / 4} I_{4}^{\prime}(G)=\frac{1}{\sqrt{2}}\left(-g^{0} g_{1} g_{2} g_{3}\right)^{1 / 4}\left(0,1 / g_{1}, 1 / g_{2}, 1 / g_{3}, \quad 0, \quad 0, \quad 0,-1 / g_{0}\right)^{T},
$$

so that we find

$$
R_{\mathrm{AdS}}=I_{4}(G)^{-1 / 4}=\left(-4 g^{0} g_{1} g_{2} g_{3}\right)^{-1 / 4},\left.\quad t^{i}\right|_{\infty}=\mathrm{i} \sqrt{-\frac{g_{1} g_{2} g_{3}}{g^{0}}} \frac{1}{g_{i}} .
$$

The above relations are not well defined for all signs of the components in $G$, so one needs to make a choice, which we take to be $g^{0}<0$ and $g_{i}>0$ throughout the discussion below.

In order to obtain the attractor solution for given charges and the full flow to the asymptotic vacuum described by (4.2)-(4.4), one needs to solve (2.11) for the vector $\mathcal{B}$. For completeness, we take the components of $\mathcal{B}$ as

$$
\mathcal{B}=\left(\beta^{0}, \quad \beta^{i}, \quad \beta_{i}, \quad \beta_{0}\right)^{T},
$$

and give explicitly the left hand side of (3.37), as

$$
\frac{1}{4} I_{4}^{\prime}(\mathcal{B}, \mathcal{B}, G)=\left(\begin{array}{c}
-g^{0} \beta^{I} \beta_{I}-\beta^{0} g^{I} \beta_{I} \\
-\beta^{i} g^{I} \beta_{I}+2 \beta^{i} \sum_{j \neq i} \beta^{j} g_{j}-c^{i j k}\left(2 \beta^{0} \beta_{j} g_{k}+g_{0} \beta_{j} \beta_{k}\right) \\
g_{i} \beta^{I} \beta_{I}+\beta_{i} g^{I} \beta_{I}-2 \beta_{i} \sum_{j \neq i} \beta^{j} g_{j}-2 g_{i} \sum_{j \neq i} \beta^{j} \beta_{j} \\
+\beta_{0} g^{I} \beta_{I}+c^{i j k} g_{i} \beta_{j} \beta_{k}
\end{array}\right),
$$

where we used the shorthand notation

$$
\beta^{I} \beta_{I}=\beta^{0} \beta_{0}+\beta^{i} \beta_{i}, \quad g^{I} \beta_{I}=g^{0} \beta_{0}+g_{i} \beta^{i},
$$

and $c^{i j k}$ is a completely symmetric tensor which is $c^{123}=1$ and and vanishes when any two indices are equal.

It is useful to note that the rescaling

$$
\begin{aligned}
\beta^{0} \rightarrow \frac{g_{0}}{\sqrt{I_{4}(G)}} \hat{\beta}^{0}, & \beta^{i} \rightarrow \frac{\hat{\beta}^{i}}{g_{i}}, & \beta_{i} \rightarrow \frac{g_{i}}{\sqrt{I_{4}(G)}} \hat{\beta}_{i}, & \beta_{0} \rightarrow \frac{\hat{\beta}_{0}}{g^{0}}, \\
p^{0} \rightarrow \frac{g_{0}}{\sqrt{I_{4}(G)} \hat{p}^{0},} & p^{i} \rightarrow \frac{\hat{p}^{i}}{g_{i}}, & q_{i} \rightarrow \frac{g_{i}}{\sqrt{I_{4}(G)}} \hat{q}_{i}, & q_{0} \rightarrow \frac{\hat{q}_{0}}{g^{0}},
\end{aligned}
$$

on the components of $\mathcal{B}$ and the charges, eliminates all explicit gaugings from (3.37) when (4.6) is used. One can therefore solve (3.37) with $I_{4}^{\prime}(\mathcal{B}, \mathcal{B}, G)$ as in (4.6) with all $g^{0}=g_{i}=1$ and re-introduce them at the end using the inverse of (4.8) for both the components of $\mathcal{B}$ and the charges. Note that this is only relevant when the gauging is in the frame (4.2), or frames obtained from it by simple enough dualities. One such example is the duality

$$
p^{0} \rightarrow p^{0}, \quad p^{i} \rightarrow q_{i}, \quad q_{i} \rightarrow-p^{i}, \quad q_{0} \rightarrow q_{0},
$$


which leads to a prepotential, $F \sim \sqrt{X^{0} X^{1} X^{2} X^{3}}$, that can be uplifted to M-theory, provided all gauging parameters are equal $[11,23]$. It follows that the expressions given in terms of the rescaled quantities (4.8) can be directly used for such an uplift, up to a possible convention dependent redefinition of the parameters. Similar (but more complicated) rescalings can be obtained in all other frames by duality, but we are not aware of any physical meaning associated to this operation.

\subsection{The $t^{3}$ model}

The first model we consider is the $t^{3}$ model, defined by the prepotential

$$
F=\frac{\left(X^{1}\right)^{3}}{X^{0}}
$$

and is very useful in building intuition for the general case, since all symmetric models can be truncated down to it. Indeed, we will view the $t^{3}$ model as the special case of the STU model for which all three scalars are equal. We therefore use the various relations (4.2)(4.6) with all indices $1,2,3$ equal. ${ }^{6}$

We now consider the most general vectors $\Gamma$ and $\mathcal{B}$ allowed by (3.38) and (3.39), given by

$$
\begin{aligned}
\mathcal{B} & =\left(\begin{array}{lll}
-\frac{3 g^{0}}{g_{1}} \frac{g^{0} \beta_{0}+g_{1} \beta^{1}}{\kappa+4 g_{1} p^{1}} q_{1}, & \beta^{1}, & \frac{g^{0} \beta_{0}+g_{1} \beta^{1}}{\kappa+4 g_{1} p^{1}} q_{1}, \quad \beta_{0}
\end{array}\right)^{T}, \\
\Gamma & =\left(\begin{array}{llll}
-\frac{3 g^{0}}{g_{1}} q_{1}, & p^{1}, & q_{1}, & \frac{1}{g^{0}}\left(\kappa+3 g_{1} p^{1}\right)
\end{array}\right)^{T},
\end{aligned}
$$

where we note that the two parameters $\beta^{1}, \beta_{0}$ are constrained by (3.41), so that there is only one independent component in $\mathcal{B}$. It follows that (3.37) can only be solved if the two charges $p^{1}, q_{1}$ are related, so that the general solution is parametrised by a single charge. One finds that there are two branches of solutions, which we now discuss in turn.

The simpler of the two branches arises by setting $q_{1}=0$, so that there is only a $p^{1}$ charge, that we take as independent, and a $q_{0}$ charge which we take to be fixed as in (4.11). The two parameters, $\beta^{1}, \beta_{0}$ read

$$
\begin{aligned}
& \beta_{0}=-\frac{1}{4 g^{0}}\left(3 \sqrt{\kappa+4 g_{1} p^{1}}+\sqrt{\kappa+12 g_{1} p^{1}}\right), \\
& \beta^{1}=\frac{1}{4 g_{1}}\left(\sqrt{\kappa+12 g_{1} p^{1}}-\sqrt{\kappa+4 g_{1} p^{1}}\right),
\end{aligned}
$$

which reproduce the result of [8-10], up to the shift (3.30). Note that there is a lower limit for $p^{1}$ for this solution to be regular, i.e. $\kappa+4 g_{1} p^{1}>0$. In this configuration, the axion is trivial throughout the flow.

\footnotetext{
${ }^{6}$ Note that this is equivalent, but not exactly identical to the $t^{3}$ model one would find directly from (4.10), since for example we use a modified inner product for which e.g. $\langle\Gamma, G\rangle=g^{0} q_{0}-3 g_{1} p^{1}$, arising from the identification of the three scalars in the STU model. These differences can be undone by appropriate rescalings, which we ignore for simplicity.
} 
The second branch allows for all charges to be nonzero, though fixed in terms of a single independent parameter. We find that the charge vector is as in (4.11) with the additional constraint

$$
q_{1}= \pm \frac{\sqrt{3}}{4 \sqrt{-g_{0} g^{1}}}\left(\kappa+4 g_{1} p^{1}\right),
$$

while the solution for the $\beta^{1}, \beta_{0}$ takes the form

$$
\begin{aligned}
& \beta_{0}=-\frac{1}{4 \sqrt{2} g^{0}}\left(4 \sqrt{-\left(\kappa+3 g_{1} p^{1}\right)}-3 \sqrt{-\left(\kappa+4 g_{1} p^{1}\right)}\right), \\
& \beta^{1}=\frac{1}{4 \sqrt{2} g_{1}}\left(4 \sqrt{-\left(\kappa+3 g_{1} p^{1}\right)}+\sqrt{-\left(\kappa+4 g_{1} p^{1}\right)}\right),
\end{aligned}
$$

for both signs in (4.13). This is similar to (4.12) but has instead the opposite behaviour with respect to the magnetic charge that is now subject to an upper bound, $\left(\kappa+3 g_{1} p^{1}\right)<0$, by regularity. Indeed, one may not turn off the electric charge and the axion continuously, unless $\kappa=-1$, which corresponds to solutions with a hyperbolic horizon.

We have checked explicitly that both (4.12) and (4.14) lead to physical solutions that satisfy all constraints considered above, in the corresponding domain for the charge $p^{1}$. In particular, they both lead to a finite area horizon consistent with (2.16) and respect (3.40). We refrain from giving the full expressions for the scalar, since they are not particularly illuminating, especially in the case of the solution with a running axion (4.14). However, we do note the interesting fact that the quartic invariant of the physical charge in (4.11) is always positive for both solutions in (4.12) and (4.14).

It is perhaps surprising to find this relatively rich set of BPS solutions, given that there is only a single complex scalar. It would be very interesting to obtain a holographic interpretation of the two solutions above, especially for the case including the axion. Presumably, one may interpret this as a broken phase controlled by an additional VEV.

\subsection{The STU model}

We now turn to the more general STU model, defined by the prepotential (4.1) which is routinely used as a benchmark example for all symmetric models in the $\mathrm{SO}(2, n+1)$ series. Using intuition from the $t^{3}$ model, we can in fact solve (4.2)-(4.6) in the case of generic charges. The result is a solution with five independent charges, which realises the two parameter duality boost described recently in [18]. Given the complexity of the general equations, we will use the rescaled variables (4.8) throughout the discussion, suppressing the explicit hat.

We first give the analogue of the solution (4.12) in the STU model, i.e. a solution with charge

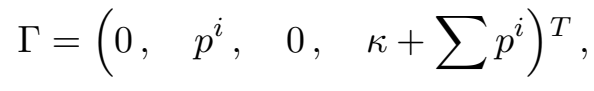

$$
\begin{aligned}
& \mathcal{B}=\left(\begin{array}{lll}
0, & \beta^{i}, \quad 0, \quad \beta_{0}
\end{array}\right)^{T},
\end{aligned}
$$

for reasons of comparison with $[8,17]$, where the same solution was given, but with a vector, $\mathcal{B}$, shifted as in (3.30), relative to the one used here. Solving (3.37) with the 
explicit expression (4.6), we obtain the components

$$
\begin{aligned}
& \beta^{i}=\frac{1}{4}\left(2 \sqrt{\Delta^{i}}-\sum_{j} \sqrt{\Delta^{j}}+\sqrt{\sum_{j} \Delta^{j}-2 \kappa}\right), \\
& \beta_{0}=-\frac{1}{4}\left(\sum_{i} \sqrt{\Delta^{i}}+\sqrt{\sum_{i} \Delta^{i}-2 \kappa}\right),
\end{aligned}
$$

where we use the quantity

$$
\Delta^{1}=\frac{\left(\kappa+2 p^{1}+2 p^{3}\right)\left(\kappa+2 p^{1}+2 p^{2}\right)}{\left(\kappa+2 p^{2}+2 p^{3}\right)},
$$

and its cyclic permutations.

We now turn to solutions including electric charges and axions, so that we consider the following vectors

$$
\begin{aligned}
& \Gamma=\left(-\sum q_{i}, \quad p^{i}, \quad q_{i}, \quad \kappa+\sum p^{i}\right)^{T} \\
& \mathcal{B}=\left(-\sum \beta_{i}, \quad \beta^{i}, \quad \beta_{i}, \quad \beta_{0}\right)^{T},,
\end{aligned}
$$

which satisfy three of the conditions (3.38)-(3.39), but do not mutually commute. We did not impose this condition yet, as it seems to complicate the equations in (3.37) in first instance. However, the condition $\langle\Gamma, \mathcal{B}\rangle=0$ imposes a constraint on the charges of the resulting solution, which turns out to be given in terms of the parameter

$$
\delta_{1}= \pm \frac{\left(q_{1}+q_{2}\right)\left(q_{1}+q_{3}\right)}{\left(\kappa+2 p^{1}+2 p^{2}\right)\left(\kappa+2 p^{1}+2 p^{3}\right)},
$$

and its cyclic permutations, $\delta_{2}, \delta_{3}$, as

$$
\frac{1}{\delta_{1}}+\frac{1}{\delta_{2}}+\frac{1}{\delta_{3}}=1
$$

Note that the two signs in the definition of the parameters $\delta_{i}$ in (4.19) lead to two branches of solutions. These relations are analogous to (4.13) and reduce to it in upon truncation to the $t^{3}$ model.

The electrically charged solutions are given in terms of the quantity

$$
\Delta^{1}=\sqrt{\frac{\left(1-\delta_{1}\right)}{\left(1-\delta_{2}\right)\left(1-\delta_{3}\right)} \frac{\left(\kappa+2 p^{1}+2 p^{3}\right)\left(\kappa+2 p^{1}+2 p^{2}\right)}{\left(\kappa+2 p^{2}+2 p^{3}\right)}},
$$

and its cyclic permutations. The components of the vector $\mathcal{B}$ are then given by

$$
\begin{aligned}
& \beta_{1}=\frac{-\left(q_{2}+q_{3}\right) \Delta^{1}+\left(q_{1}+q_{3}\right) \Delta^{2}+\left(q_{1}+q_{2}\right) \Delta^{3}}{4 \Delta^{1} \Delta^{2} \Delta^{3}} \sum_{i} \Delta^{i}-\frac{\left(2 q_{1}+q_{3}+q_{2}\right)}{2 \Delta^{1}}, \\
& \beta^{i}=\frac{1}{4}\left(2 \Delta^{i}-\sum_{i} \Delta^{i}+\Delta^{0}\right)
\end{aligned}
$$




$$
\begin{aligned}
& \beta_{0}=-\frac{1}{4}\left(\sum_{i} \Delta^{i}+\sqrt{\Delta^{0}}\right), \\
& \Delta^{0}=16\left(\sum_{i} \Delta^{i}\right)^{2}-4\left(\beta_{1} \beta_{2}+\beta_{1} \beta_{3}+\beta_{2} \beta_{3}\right)-8\left(\kappa+p^{1}+p^{2}+p^{3}\right),
\end{aligned}
$$

where $\beta_{2}$ and $\beta_{3}$ are given by the obvious cyclic permutations of $\beta_{1}$. Once again, the truncation to the $t^{3}$ model leads to the expression (4.14) and we find that all the requirements for a physical solution are satisfied if the $p^{i}<0$. We refrain from giving further explicit expressions for the scalars, as they are not particularly illuminating.

\section{Conclusion and outlook}

In this paper, we have considered the general static 1/4-BPS flow in Fayet-Iliopoulos gauged $\mathcal{N}=2$ supergravity coupled to vector multiplets describing a symmetric scalar manifold. Using techniques from very special geometry, we have shown that it is possible to reduce the BPS equations to a form where the scalar fields only appear through a single combination that transforms covariantly under electric/magnetic duality reparametrisations. Considering a general expansion at the horizon, we have found that physical black hole solutions are very restricted, their basic properties being captured by the standard ansatz that was proposed for the simplified axion-free case [8-10, 17, 18].

In addition, we have found that the full flow is parametrised in terms of a real symplectic vector that is determined by the gauging and charge vectors and can moreover be identified with the solution to the attractor equations. The final result is a set of algebraic equations at the attractor, whose solution implies the existence of a full asymptotically $\mathrm{AdS}_{4}$ black hole solution. These equations involve the quartic invariant of very special geometry taking as arguments both the charges and gaugings, in a way similar to the two-charge invariants of [24-26]. We have given a number of explicit examples of solutions to these equations, describing black holes with all allowed electric and magnetic charges turned on, as well as nontrivial axions, in the STU model and its truncations.

In view of the relative complexity of the original flow equations, this is a somewhat surprising result. For example, the Kähler connection appears to be necessarily trivial, despite the fact that the BPS equations do not impose that requirement directly. Instead, demanding regularity of black hole solutions one finds that the corresponding BPS equation must be trivially satisfied, with the terms involved vanishing individually. However, we expect this to change in the case of rotating and/or NUT-charged black holes $[11,13]$. Indeed, this was shown to be true for the asymptotically flat solutions to the theory in [20], where the scalar flow of the asymptotically flat extremal non-BPS black holes [27] was shown to be very similar to the one studied in this paper.

It is important to note that the method used to simplify the BPS flow equations in this paper is straightforward to apply in more general situations, for example in general stationary solutions and in theories involving hypermultiplets, as long as the vector multiplet scalars are parametrising a symmetric manifold. The latter observation is particularly important, given that, in a general gauged theory, the BPS flow for the vector multiplet 
scalars depends on the hyperscalars only through the moment maps, arranged in a symplectic vector, $\left(P^{I}, P_{I}\right)^{T}$, which generalises the vector of FI parameters, $G$, considered in this paper. Since we did not make any use of the fact that $G$ is constant in the derivation of the simplified flow equation, it follows that one may repeat exactly the same steps to simplify the general BPS conditions. Finally, it is interesting to consider the possibility of extending the ansatze proposed for axion-free non-BPS solutions to FI gauged supergravity [28-31] to the general case, using the same method. While the systems mentioned above are considerably more complicated, we expect that the steps followed for the system studied in this paper will still be be useful. We hope to return to some of these issues in the future.

\section{Acknowledgments}

The author wishes to thank Kiril Hristov for introducing him to this subject through many valuable discussions and for comments on an earlier version of this paper. He further thanks Nick Halmagyi for discussions and critical comments on an earlier version of this paper. Further useful discussions with Guillaume Bossard and Alessandro Tomasiello are gratefully acknowledged. The work of S.K. is supported by the European Research Council under the European Union's Seventh Framework Program (FP/2007-2013)-ERC Grant Agreement n. 307286 (XD-STRING). His research is also supported in part by INFN.

\section{A Conventions on $\mathcal{N}=2$ supergravity}

In this paper we follow the notation and conventions of [32]. In this appendix we collect some basic definitions that are useful in the main text, referring to that paper for more details.

The vector fields naturally arrange in a symplectic vector of electric and magnetic gauge field strengths, whose integral over a sphere defines the associated electromagnetic charges as

$$
\mathcal{F}_{\mu \nu}=\left(\begin{array}{c}
F_{\mu \nu}^{I} \\
G_{I \mu \nu}
\end{array}\right), \quad \Gamma=\left(\begin{array}{c}
p^{I} \\
q_{I}
\end{array}\right)=\frac{1}{2 \pi} \int_{S^{2}} \mathcal{F} .
$$

Here, $F_{\mu \nu}^{I}$ are the field strengths of the vector fields, while the $G_{I \mu \nu}$ stand for the dual field strengths defined by taking a derivative of the Lagrangian or, equivalently, by the scalar dependent period matrix, $\mathcal{N}_{I J}$, as

$$
G_{\mu \nu I}^{-}=\mathcal{N}_{I J} F_{\mu \nu}^{-J}
$$

where the explicit form of the period matrix will not be used.

The physical scalar fields $t^{i}$, which parametrize a special Kähler space of complex dimension $n_{\mathrm{v}}$, appear through the so called symplectic section, $\mathcal{V}$. Choosing a basis, this section can be written in components in terms of scalars $X^{I}$ as

$$
\mathcal{V}=\left(\begin{array}{c}
X^{I} \\
F_{I}
\end{array}\right), \quad F_{I}=\frac{\partial F}{\partial X^{I}},
$$


where $F$ is a holomorphic function of degree two, called the prepotential, which we will always consider to be cubic

$$
F=-\frac{1}{6} c_{i j k} \frac{X^{i} X^{j} X^{k}}{X^{0}},
$$

for completely symmetric $c_{i j k}, i=1, \ldots n_{\mathrm{v}}$. The section $\mathcal{V}$ is subject to the constraints

$$
\langle\overline{\mathcal{V}}, \mathcal{V}\rangle=i \quad\left\langle\bar{D}_{\bar{i}} \overline{\mathcal{V}}, D_{j} \mathcal{V}\right\rangle=-i g_{i j}
$$

with all other inner products vanishing, and is uniquely determined by the physical scalar fields $t^{i}=\frac{X^{i}}{X^{0}}$ up to a local U(1) transformation. Here, $g_{\bar{\imath} j}$ is the Kähler metric and the Kähler covariant derivative $D_{i} \mathcal{V}$ contains the Kähler connection $Q_{\mu}$, defined through the Kähler potential as

$$
Q=\operatorname{Im}\left[\partial_{i} \mathcal{K} d t^{i}\right], \quad \mathcal{K}=-\ln \left(\frac{i}{6} c_{i j k}(t-\bar{t})^{i}(t-\bar{t})^{j}(t-\bar{t})^{k}\right)
$$

We introduce the following notation for any symplectic vector $\Gamma$

$$
Z(\Gamma)=\langle\Gamma, \mathcal{V}\rangle, \quad Z_{i}(\Gamma)=\left\langle\Gamma, D_{i} \mathcal{V}\right\rangle,
$$

with the understanding that when an argument does not appear explicitly, the vector of charges in (A.1) should be inserted. In addition, when the argument is form valued, the operation is applied component wise. With these definitions it is possible to introduce a scalar dependent complex basis for symplectic vectors, given by $\left(\mathcal{V}, D_{i} \mathcal{V}\right)$, so that any vector $\Gamma$ can be expanded as

$$
\Gamma=2 \operatorname{Im}\left[-\bar{Z}(\Gamma) \mathcal{V}+g^{\bar{\imath} j} \bar{Z}_{\bar{\imath}}(\Gamma) D_{j} \mathcal{V}\right]
$$

whereas the symplectic inner product can be expressed as

$$
\left\langle\Gamma_{1}, \Gamma_{2}\right\rangle=2 \operatorname{Im}\left[-Z\left(\Gamma_{1}\right) \bar{Z}\left(\Gamma_{2}\right)+g^{i \bar{\jmath}} Z_{i}\left(\Gamma_{1}\right) \bar{Z}_{\bar{\jmath}}\left(\Gamma_{2}\right)\right] .
$$

In addition, we introduce the scalar dependent complex structure $\mathrm{J}$, defined as

$$
\mathrm{J} \mathcal{V}=-i \mathcal{V}, \quad \mathrm{J} D_{i} \mathcal{V}=i D_{i} \mathcal{V}
$$

which can be solved to determine $J$ in terms of the period matrix $\mathcal{N}_{I J}$ in (A.2), see e.g. [33] for more details. With this definition, we can express the complex self-duality of the gauge field strengths as

$$
\mathrm{J} \mathcal{F}=-* \mathcal{F},
$$

which is the duality covariant form of the relation between electric and magnetic components. Finally, we record the important relation

$$
\frac{1}{2}\langle\Gamma, \mathrm{J} \Gamma\rangle=|Z(\Gamma)|^{2}+g^{i \bar{\jmath}} Z_{i}(\Gamma) \bar{Z}_{\bar{\jmath}}(\Gamma) \equiv V_{\mathrm{BH}}(\Gamma),
$$

where we defined the black hole potential $V_{\mathrm{BH}}(\Gamma)$. 


\section{B Identities involving the quartic invariant}

In this short appendix, we summarise a number of useful relations involving the quartic invariant, defined for all symmetric models. The starting point is the definition of the invariant, $I_{4}(\Gamma)$, for any symplectic vector, $\Gamma$, as $[34,35]$

$$
\begin{aligned}
I_{4}(\Gamma) & =\frac{1}{4 !} t^{M N P Q} \Gamma_{M} \Gamma_{N} \Gamma_{P} \Gamma_{Q} \\
& =-\left(p^{0} q_{0}+p^{i} q_{i}\right)^{2}+\frac{2}{3} q_{0} c_{i j k} p^{i} p^{j} p^{k}-\frac{2}{3} p^{0} c^{i j k} q_{i} q_{j} q_{k}+c_{i j k} p^{j} p^{k} c^{i l m} q_{l} q_{m},
\end{aligned}
$$

where $M, N \ldots$ are indices encompassing both electric and magnetic components and we also defined the completely symmetric tensor $t^{M N P Q}$ for later reference. It is also convenient to define a symplectic vector out the first derivative, $I_{4}^{\prime}(\Gamma)$, of the quartic invariant, as

$$
I_{4}^{\prime}(\Gamma)_{M} \equiv \Omega_{M N} \frac{\partial I_{4}(\Gamma)}{\partial \Gamma_{N}}=\frac{1}{3 !} \Omega_{M N} t^{N P Q R} \Gamma_{P} \Gamma_{Q} \Gamma_{R},
$$

where $\Omega^{M N}$ is the symplectic form, so that the following relations hold

$$
\left\langle\Gamma, I_{4}^{\prime}(\Gamma)\right\rangle=4 I_{4}(\Gamma), \quad I_{4}^{\prime}(\Gamma, \Gamma, \Gamma)=6 I_{4}^{\prime}(\Gamma) .
$$

Throughout this paper, all instances of $I_{4}\left(\Gamma_{1}, \Gamma_{2}, \Gamma_{3}, \Gamma_{4}\right)$ will denote the contraction of the tensor $t^{M N P Q}$ in (B.1) with the four charges, without any symmetry factors, except for the case with a single argument, as in $I_{4}(\Gamma)$ and $I_{4}^{\prime}(\Gamma)$. For more details on this tensor, see [27] in the real basis and [36] in the complex basis, to be defined shortly.

We now record some identities that are used repeatedly in the main text, starting with the fundamental property

$$
I_{4}^{\prime}\left(I_{4}^{\prime}(\Gamma)\right)=-16 I_{4}(\Gamma)^{2} \Gamma .
$$

Further properties include the septic and quintic identities

$$
I_{4}^{\prime}\left(I_{4}^{\prime}(\Gamma), I_{4}^{\prime}(\Gamma), \Gamma\right)=8 I_{4}(\Gamma) I_{4}^{\prime}(\Gamma), \quad I_{4}^{\prime}\left(I_{4}^{\prime}(\Gamma), \Gamma, \Gamma\right)=-8 I_{4}(\Gamma) \Gamma .
$$

Finally, the projection operator

$$
I_{4}^{\prime}\left(I_{4}^{\prime}(\Gamma), \Gamma, \mathcal{B}\right)=2\langle\Gamma, \mathcal{B}\rangle I_{4}^{\prime}(\Gamma)+2\left\langle I_{4}^{\prime}(\Gamma), \mathcal{B}\right\rangle \Gamma,
$$

where $\mathcal{B}$ is an arbitrary vector, is particularly useful in the analysis of sections 2 and 3.

One can rewrite the quartic invariant in the complex basis [37], leading to the following alternative definition

$$
I_{4}(\Gamma)=\left(Z \bar{Z}-Z_{i} \bar{Z}^{i}\right)^{2}-c_{m i j} \bar{Z}^{i} \bar{Z}^{j} c^{m k l} Z_{k} Z_{l}+\frac{2}{3} \bar{Z} c^{i j k} Z^{i} Z^{j} Z^{k}+\frac{2}{3} Z c_{i j k} \bar{Z}^{i} \bar{Z}^{j} \bar{Z}^{k} .
$$

Despite the appearance of the central charges, this expression is by construction independent of the scalars, which only appear due to the change of basis in (A.8). The derivatives of (B.7) with respect to the central charges $Z(\Gamma)$ and $Z_{i}(\Gamma)$ can be used to define the tensor $t^{M N P Q}$ and its contractions in the complex basis, in exactly the same way as above. We will not make use of this basis, but we do note an identity central to the analysis of 
sections 2 and 3. Consider the contraction of (B.7) with two instances of a charge and two instances of the symplectic section itself, so that the resulting expression is at most quadratic in the central charges $Z(\Gamma)$ and $Z_{i}(\Gamma)$, due to (A.5). The resulting equality reads

$$
\frac{1}{4} I_{4}(\Gamma, \Gamma, 2 \operatorname{Im} \mathcal{V}, 2 \operatorname{Im} \mathcal{V})=4\left(|Z(\Gamma)|^{2}+\operatorname{Re}(Z(\Gamma))^{2}\right)-2\left(|Z(\Gamma)|^{2}+Z_{i}(\Gamma) \bar{Z}(\Gamma)^{i}\right),
$$

while its derivative is

$$
\frac{1}{2} I_{4}(\Gamma, 2 \operatorname{Im} \mathcal{V}, 2 \operatorname{Im} \mathcal{V})=8 \operatorname{Im}(Z(\Gamma)) \operatorname{Im} \mathcal{V}+16 \operatorname{Re}(Z(\Gamma)) \operatorname{Re} \mathcal{V}-2 \mathrm{~J} \Gamma .
$$

The last equation relates the action of the scalar dependent complex structure on the charge to a matrix operation involving the quartic invariant. Since the quartic invariant is evaluated with two instances of the symplectic section, this form is particularly useful in solving the BPS equations for black hole backgrounds, where an ansatz for the imaginary part of the section is usually considered.

Open Access. This article is distributed under the terms of the Creative Commons Attribution License (CC-BY 4.0), which permits any use, distribution and reproduction in any medium, provided the original author(s) and source are credited.

\section{References}

[1] K. Behrndt, D. Lüst and W.A. Sabra, Stationary solutions of $N=2$ supergravity, Nucl. Phys. B 510 (1998) 264 [hep-th/9705169] [INSPIRE].

[2] F. Denef, Supergravity flows and D-brane stability, JHEP 08 (2000) 050 [hep-th/0005049] [INSPIRE].

[3] J.P. Gauntlett, J.B. Gutowski, C.M. Hull, S. Pakis and H.S. Reall, All supersymmetric solutions of minimal supergravity in five- dimensions, Class. Quant. Grav. 20 (2003) 4587 [hep-th/0209114] [INSPIRE].

[4] J.P. Gauntlett and J.B. Gutowski, General concentric black rings, Phys. Rev. D 71 (2005) 045002 [hep-th/0408122] [INSPIRE].

[5] S.L. Cacciatori, D. Klemm, D.S. Mansi and E. Zorzan, All timelike supersymmetric solutions of $N=2, D=4$ gauged supergravity coupled to abelian vector multiplets, JHEP 05 (2008) 097 [arXiv: 0804.0009] [INSPIRE].

[6] D. Klemm and E. Zorzan, The timelike half-supersymmetric backgrounds of $N=2, D=4$ supergravity with Fayet-Iliopoulos gauging, Phys. Rev. D 82 (2010) 045012 [arXiv: 1003.2974] [INSPIRE].

[7] P. Meessen and T. Ortín, Supersymmetric solutions to gauged $N=2 D=4$ SUGRA: the full timelike shebang, Nucl. Phys. B 863 (2012) 65 [arXiv:1204.0493] [INSPIRE].

[8] S.L. Cacciatori and D. Klemm, Supersymmetric AdS 4 black holes and attractors, JHEP 01 (2010) 085 [arXiv: 0911.4926] [INSPIRE].

[9] G. Dall'Agata and A. Gnecchi, Flow equations and attractors for black holes in $N=2 U(1)$ gauged supergravity, JHEP 03 (2011) 037 [arXiv: 1012.3756] [INSPIRE].

[10] K. Hristov and S. Vandoren, Static supersymmetric black holes in $A d S_{4}$ with spherical symmetry, JHEP 04 (2011) 047 [arXiv: 1012.4314] [INSPIRE]. 
[11] D. Klemm, Rotating BPS black holes in matter-coupled AdS $S_{4}$ supergravity, JHEP 07 (2011) 019 [arXiv: 1103.4699] [INSPIRE].

[12] S. Barisch, G. Lopes Cardoso, M. Haack, S. Nampuri and N.A. Obers, Nernst branes in gauged supergravity, JHEP 11 (2011) 090 [arXiv:1108.0296] [INSPIRE].

[13] M. Colleoni and D. Klemm, Nut-charged black holes in matter-coupled $N=2, D=4$ gauged supergravity, Phys. Rev. D 85 (2012) 126003 [arXiv:1203.6179] [InSPIRE].

[14] N. Halmagyi, M. Petrini and A. Zaffaroni, BPS black holes in AdS $S_{4}$ from M-theory, JHEP 08 (2013) 124 [arXiv: 1305.0730] [INSPIRE].

[15] N. Halmagyi, BPS Black Hole Horizons in $N=2$ Gauged Supergravity, JHEP 02 (2014) 051 [arXiv: 1308.1439] [INSPIRE].

[16] S. Barisch-Dick, G.L. Cardoso, M. Haack and Á. Véliz-Osorio, Quantum corrections to extremal black brane solutions, JHEP 02 (2014) 105 [arXiv:1311.3136] [INSPIRE].

[17] A. Gnecchi and N. Halmagyi, Supersymmetric black holes in AdS $S_{4}$ from very special geometry, JHEP 04 (2014) 173 [arXiv:1312.2766] [INSPIRE].

[18] N. Halmagyi and T. Vanel, AdS Black Holes from Duality in Gauged Supergravity, JHEP 04 (2014) 130 [arXiv:1312.5430] [INSPIRE].

[19] S. Bellucci, S. Ferrara, A. Marrani and A. Yeranyan, $D=4$ Black Hole Attractors in $N=2$ Supergravity with Fayet-Iliopoulos Terms, Phys. Rev. D 77 (2008) 085027 [arXiv:0802.0141] [INSPIRE].

[20] K. Hristov, S. Katmadas and V. Pozzoli, Ungauging black holes and hidden supercharges, JHEP 01 (2013) 110 [arXiv:1211.0035] [INSPIRE].

[21] D.D.K. Chow and G. G. Compère, Dyonic AdS black holes in maximal gauged supergravity, Phys. Rev. D 89 (2014) 065003 [arXiv:1311.1204] [InSPIRE].

[22] A. Gnecchi, K. Hristov, D. Klemm, C. Toldo and O. Vaughan, Rotating black holes in $4 d$ gauged supergravity, JHEP 01 (2014) 127 [arXiv:1311.1795] [INSPIRE].

[23] M. Cvetič, M.J. Duff, P. Hoxha, J.T. Liu, H. Lü et al., Embedding AdS black holes in ten-dimensions and eleven-dimensions, Nucl. Phys. B 558 (1999) 96 [hep-th/9903214] [INSPIRE].

[24] S. Ferrara, A. Marrani, E. Orazi, R. Stora and A. Yeranyan, Two-Center Black Holes Duality-Invariants for STU Model and its lower-rank Descendants, J. Math. Phys. 52 (2011) 062302 [arXiv: 1011.5864] [INSPIRE].

[25] L. Andrianopoli, R. D'Auria, S. Ferrara, A. Marrani and M. Trigiante, Two-Centered Magical Charge Orbits, JHEP 04 (2011) 041 [arXiv:1101.3496] [INSPIRE].

[26] A. Ceresole, S. Ferrara, A. Marrani and A. Yeranyan, Small Black Hole Constituents and Horizontal Symmetry, JHEP 06 (2011) 078 [arXiv:1104.4652] [INSPIRE].

[27] G. Bossard and S. Katmadas, Duality covariant multi-centre black hole systems, JHEP 08 (2013) 007 [arXiv: 1304.6582] [INSPIRE].

[28] D. Klemm and O. Vaughan, Nonextremal black holes in gauged supergravity and the real formulation of special geometry, JHEP 01 (2013) 053 [arXiv:1207.2679] [INSPIRE].

[29] C. Toldo and S. Vandoren, Static nonextremal AdS4 black hole solutions, JHEP 09 (2012) 048 [arXiv: 1207.3014] [INSPIRE]. 
[30] D. Klemm and O. Vaughan, Nonextremal black holes in gauged supergravity and the real formulation of special geometry II, Class. Quant. Grav. 30 (2013) 065003 [arXiv:1211.1618] [INSPIRE].

[31] A. Gnecchi and C. Toldo, On the non-BPS first order flow in $N=2$ U(1)-gauged Supergravity, JHEP 03 (2013) 088 [arXiv:1211.1966] [INSPIRE].

[32] G. Bossard and S. Katmadas, Duality covariant non-BPS first order systems, JHEP 09 (2012) 100 [arXiv: 1205.5461] [INSPIRE].

[33] A. Ceresole, R. D'Auria and S. Ferrara, The Symplectic structure of $N=2$ supergravity and its central extension, Nucl. Phys. Proc. Suppl. 46 (1996) 67 [hep-th/9509160] [InSPIRE].

[34] S. Ferrara and M. Günaydin, Orbits of exceptional groups, duality and BPS states in string theory, Int. J. Mod. Phys. A 13 (1998) 2075 [hep-th/9708025] [INSPIRE].

[35] S. Ferrara, E.G. Gimon and R. Kallosh, Magic supergravities, $N=8$ and black hole composites, Phys. Rev. D 74 (2006) 125018 [hep-th/0606211] [INSPIRE].

[36] S. Ferrara, A. Marrani and A. Yeranyan, On Invariant Structures of Black Hole Charges, JHEP 02 (2012) 071 [arXiv:1110.4004] [INSPIRE].

[37] B.L. Cerchiai, S. Ferrara, A. Marrani and B. Zumino, Duality, Entropy and ADM Mass in Supergravity, Phys. Rev. D 79 (2009) 125010 [arXiv:0902.3973] [INSPIRE]. 